\title{
Falkner-Skan Flow over a Wedge with Slip Boundary Conditions
}

\author{
Michael J. Martin* and Iain D. Boyd \\ University of Michigan, Ann Arbor, Michigan 48109-2140
}

DOI: $\underline{10.2514 / 1.43316}$

\begin{abstract}
The Falkner-Skan solution for laminar boundary-layer flow over a wedge is modified to allow for a slip boundary condition. A modified boundary-layer Knudsen number $K$ is introduced, and the coordinate system is transformed from one-dimensional to two-dimensional to allow for the loss of self-similarity in the flow. A marching scheme is used to solve the boundary-layer equations in the rarefied flow regime. The results of this solution show decreased skin friction, boundary-layer thickness, velocity thickness, and momentum thickness because of the presence of the slip boundary condition. When the energy equation is solved using a temperature-jump boundary condition, the heat transfer increases for slightly rarefied flows, and then decreases as the Knudsen number increases.
\end{abstract}

\section{Nomenclature}

$\begin{array}{lll}b & = & \text { velocity coefficient } \\ c_{p} & = & \text { specific heat } \\ f & = & \text { nondimensional stream function } \\ K & = & \text { nonequilibrium parameter } \\ K n & = & \text { Knudsen number } \\ k & = & \text { thermal conductivity } \\ l & = & \text { slip length } \\ M & = & \text { Mach number } \\ m & = & \text { flow exponent } \\ n & = & \text { distance in the normal direction } \\ P & = & \text { pressure } \\ P r & = & \text { Prandtl number } \\ R e & = & \text { Reynolds number } \\ T & = & \text { temperature } \\ U & = & \text { external } x \text { velocity } \\ u & = & x \text { velocity } \\ v & = & y \text { velocity } \\ x & = & \text { position in the flow direction } \\ y & = & \text { position in the flow normal direction } \\ \alpha & = & \text { thermal diffusivity } \\ \beta & = & \text { included angle } \\ \gamma & = & \text { specific heat ratio } \\ \delta & = & \text { boundary-layer thickness } \\ \delta^{*} & = & \text { displacement thickness } \\ \eta & = & \text { nondimensional position } \\ \theta & = & \text { momentum thickness } \\ \lambda & = & \text { mean-free path } \\ \mu & = & \text { viscosity } \\ \rho & = & \text { density } \\ \sigma & = & \text { accommodation coefficient } \\ \tau & = & \text { shear stress } \\ v & = & \text { kinematic viscosity } \\ & & \end{array}$

Presented as Paper 476 at the 2009 AIAA Aerospace Sciences Meeting, Orlando, FL, 5-9 January 2008; received 19 January 2009; revision received 5 October 2009; accepted for publication 25 October 2009. Copyright $\odot 2009$ by Michael James Martin. Published by the American Institute of Aeronautics and Astronautics, Inc., with permission. Copies of this paper may be made for personal or internal use, on condition that the copier pay the $\$ 10.00$ per-copy fee to the Copyright Clearance Center, Inc., 222 Rosewood Drive, Danvers, MA 01923; include the code 0887-8722/10 and $\$ 10.00$ in correspondence with the CCC.

${ }^{*}$ Graduate Student Research Assistant, Department of Aerospace Engineering; current: Department of Mechanical Engineering, Louisiana State University, Baton Rouge, LA 70803. Member AIAA.

${ }^{\dagger}$ Professor, Department of Aerospace Engineering. Associate Fellow AIAA.

$$
\begin{array}{lll}
\text { Subscripts } & \\
g & = & \text { gas } \\
M & = & \text { momentum } \\
o & = & \text { freestream } \\
\text { slip } & = & \text { slip } \\
T & = & \text { thermal } \\
w & = & \text { wall }
\end{array}
$$

Superscript

$* \quad=$ nondimensional

\section{Introduction}

$\mathbf{T}$ HE solution of the incompressible laminar boundary-layer equations under rarefied flow conditions at low Mach numbers has applications to several areas of engineering interest, including aerosol science [1] , subsonic flight in extraterrestrial atmospheres [2], and micro and nano air vehicles $[3,4]$. Early attempts to solve the boundary-layer equations with a slip boundary condition analytically or using perturbation methods yielded a variety of results. Results assuming that the slip solution was a perturbation of the no-slip solution predicted that the slip condition would not affect shear stress, boundary-layer thickness, or heat transfer [5,6]. Additional semi-analytic results suggested that the heat transfer would change in the presence of slip flow [7-9]. Additional computations showed that the shear stress would change as well $[10,11]$.

Several explanations were offered for the contradictory results. The solutions to other viscous flows considered similar to boundarylayer flows, such as Couette, Poiseuille, and Rayleigh flows, showed a change in heat transfer and shear stress [12]. This led to the suggestion that the mathematical and experimental techniques available at the time lacked the accuracy necessary to capture the result.

The suggestion was also made that the boundary-layer equations were not valid for slip flows. Two separate arguments were made. The first was that the second-order slip boundary condition was of the same order as the terms that were discarded from the Navier-Stokes equations to create the boundary-layer equations [13,14]. A second problem was the Reynolds number scaling of the boundary-layer equations. Using the definitions of viscosity and the speed of sound, the Knudsen number can be found as a function of the Mach number and Reynolds number [15]:

$$
K n_{x} \propto M / R e_{x}
$$

This scaling indicates that an incompressible boundary layer, with a Reynolds number of 500 or greater and a Mach number of less than 
0.3 , is unlikely to have a Knudsen number large enough for slip to appear.

Several decades after these initial results, the development of microelectromechanical systems led to a renewed interest in slip flows $[16,17]$. The correct scaling of boundary-layer slip was shown to be based on the boundary-layer thickness and was computed as

$$
K n_{\delta} \propto M / \sqrt{R e_{x}}
$$

This scaling does allow an incompressible boundary layer with a Reynolds number of 500 or greater and a Mach number of less than 0.3 to have a large enough Knudsen number for slip to appear.

Complete numerical solutions of the Blasius boundary-layer equations with slip flow over a flat plate [18] contradicted the conclusion that slip did not change shear stress within a laminar boundary layer. This analysis showed that the slip condition changes the boundary layer structure from a self-similar profile to a twodimensional structure, which can be nondimensionalized using a boundary-layer Knudsen number. This solution showed decreased shear stress, boundary-layer thickness, and heat transfer. The discrepancy between full numerical results, and those obtained by using perturbation methods, suggests that the perturbation method assumed a smaller change than what appeared in the physical problem. A second possible explanation for the discrepancy between the two sets of results is that the formulation of the boundary-layer equations used in the perturbation method did not account for the loss of self-similarity in the flow.

These results also show that the assumption that the slip effects were inherently smaller than the discarded boundary-layer terms was not justified by the numeric results: If they were truly negligible, the results would be identical if they were included or not. The loss of self-similarity was consistent with trends observed in other rarefied flows, such as slip flow in microchannels $[19,20]$. Similar results were seen for the solution of related flows, such as stagnation-point flow [21-23] and the boundary layer in free convection [24].

The Blasius boundary-layer solution [25] is only one member of a larger family of boundary-layer solutions known as Falkner-Skan flow $[26,27]$. The boundary-layer equations for Falkner-Skan flow apply to two-dimensional flow over a wedge with an included angle of $\beta \pi$ and an external pressure gradient based on the inviscid flow solution. A value of $\beta$ of zero corresponds to the flat-plate Blasius solution, and a value of 1 corresponds to a stagnation region.

The present work adds a slip-flow boundary condition to the Falkner-Skan equations, allowing the fluid flow and heat transfer to be determined for a wedge in moderately rarefied flow. The boundary-layer momentum and energy equations for slip flow over a wedge are derived. A marching scheme to solve these equations is outlined. Numeric solutions are provided for flow and heat transfer over several wedge half-angles with discussion of these results.

\section{Formulation of the Governing Equations}

Because of the loss of self-similarity, the nondimensional $x$ momentum and thermal transport equations must be reformulated to incorporate variation in the $x$ direction. This requires extending nondimensionalizations previously used for the flat plate in slip flow [18] to incorporate wedge flow.

\section{A. Momentum Equation}

As shown in Fig. 1, flow over the top of a wedge can be modeled as an external flow $U(\bar{x})$ with a pressure gradient given by the inviscid flow solution. The angle of the wedge is given as $\beta \pi$.

The external flow velocity and pressure gradients are given by

$$
\begin{gathered}
U(x)=b x^{m} \\
\frac{\partial P}{\partial x}=-\rho U(x) \frac{\mathrm{d} U(x)}{\mathrm{d} x}=-m \rho b^{2} x^{2 m-1}
\end{gathered}
$$

where $U$ is the external velocity, $P$ is the pressure, $\rho$ is the density, and $x$ is the position along the wedge. The coefficient $b$ is a function of the

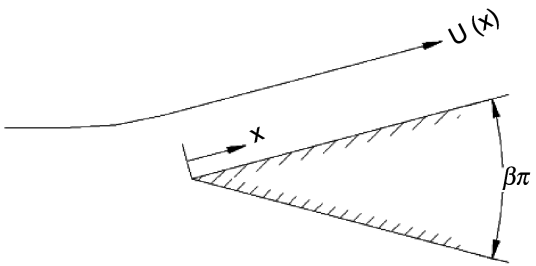

Fig. 1 Boundary-layer flow over a wedge.

flow geometry. As long as the boundary layer is relatively thin, the external flow, and the pressure gradient will be independent of the thickness of the boundary layer.

The exponent $m$ is a function of the angle $\beta$ :

$$
m=\frac{\beta}{2-\beta}
$$

The flow near the wedge will be governed by the boundary-layer equations. The equation for continuity is identical to the flat-plate case:

$$
\frac{\partial u}{\partial x}+\frac{\partial v}{\partial y}=0
$$

For steady flow in a boundary layer, the $x$-momentum equation is given by

$$
u \frac{\partial u}{\partial x}+v \frac{\partial u}{\partial y}=-\frac{1}{\rho} \frac{\mathrm{d} P(x)}{\mathrm{d} x}+v \frac{\partial^{2} u}{\partial y^{2}}
$$

where $u$ is the $x$ velocity, $v$ is the $y$ velocity, and $v$ is the kinematic viscosity.

These equations can then be transformed, using the nondimensionalizations and nondimensional stream functions developed by Falkner and Skan. These nondimensionalizations are similar to, but not identical to, those used by Blasius. A nondimensional flow coordinate $\eta$ is formed by combining $x$ and $y$ with the other flow variables:

$$
\eta=y \sqrt{\frac{m+1}{2} \frac{b}{v}} x^{\frac{m-1}{2}}
$$

A nondimensional stream function $f(\eta)$ is found from the dimensional stream function $\psi$ :

$$
\psi(x, y)=\sqrt{\frac{2 b v}{m+1}} x^{\frac{m+1}{2}} f(\eta)
$$

The nondimensional velocities are given as

$$
\begin{gathered}
u^{*}=\frac{u}{U(x)}=f^{\prime}(\eta) \\
v^{*}=\frac{v}{\sqrt{\frac{2}{m+1} v b x^{m-1}}}=\left[f(\eta)-\frac{m-1}{m+1} \eta f^{\prime}(\eta)\right]
\end{gathered}
$$

A governing equation for $f$ can be found by substituting these nondimensional terms into the $x$-momentum equation (7):

$$
f^{\prime \prime \prime}(\eta)+f(\eta) f^{\prime \prime}(\eta)+\beta\left(1-\left(f^{\prime}(\eta)\right)^{2}\right)=0
$$

For the no-slip case, the boundary conditions are

$$
\begin{gathered}
u^{*}(y=0)=0 \Rightarrow f^{\prime}(\eta=0)=0 \\
v^{*}(y=0)=0 \Rightarrow f(\eta=0)=0 \\
u^{*}(y \rightarrow \infty)=1 \Rightarrow f^{\prime}(\eta \rightarrow \infty)=1
\end{gathered}
$$


For slightly rarefied flows, the no-slip boundary condition (13) is replaced by a slip condition [료];

$$
u_{\text {slip }}=u_{g}-u_{w}=\left.\lambda \frac{2-\sigma_{M}}{\sigma_{M}} \frac{\partial u}{\partial n}\right|_{\text {wall }}+\left.\frac{3}{4} \frac{v}{T_{g}} \frac{\partial T}{\partial s}\right|_{\text {wall }}
$$

where $u_{\text {slip }}$ is the wall-slip velocity, $u_{g}$ is the gas velocity at the wall, $u_{w}$ is the wall velocity, $\partial u / \partial n$ is the velocity gradient normal to the wall, $\lambda$ is the mean-free path of the gas, $\sigma_{M}$ is the tangential momentum accommodation coefficient, $\rho$ is the density of the gas, $T_{g}$ is the temperature of the gas, and $\partial T / \partial s$ is the temperature gradient along the wall.

For an isothermal wall, Eq. (16) can be nondimensionalized to obtain

$$
\left.\frac{\partial f}{\partial \eta}\right|_{\eta=0}=\left.\frac{\left(2-\sigma_{M}\right)}{\sigma_{M}} \sqrt{\frac{m+1}{2}} \frac{\lambda}{x} \sqrt{\frac{x U(x)}{v}} \frac{\partial^{2} f}{\partial \eta^{2}}\right|_{\eta=0}=\left.K \frac{\partial^{2} f}{\partial \eta^{2}}\right|_{\eta=0}
$$

The nonequilibrium parameter $K$, which is proportional to the boundary-layer Knudsen number, is defined as

$$
\begin{aligned}
K & =\frac{\left(2-\sigma_{M}\right)}{\sigma_{M}} \sqrt{\frac{m+1}{2}} \frac{\lambda}{x} \sqrt{\frac{x U(x)}{v}} \\
& =\frac{\left(2-\sigma_{M}\right)}{\sigma_{M}} \sqrt{\frac{m+1}{2}} \lambda \sqrt{\frac{b}{v} x^{\frac{m-1}{2}}} \\
& =\frac{\left(2-\sigma_{M}\right)}{\sigma_{M}} \sqrt{\frac{m+1}{2}} K n_{x} R e_{x}^{1 / 2}
\end{aligned}
$$

where $K n_{x}$ and $R e_{x}$ are the Knudsen and Reynolds numbers based on $x$.

Just as in the flat-plate case, the revised boundary condition leads to a loss of self-similarity, and the velocity will be a function of both $\eta$ and $K$. The definition of $u^{*}$ is unchanged. The definition of $v^{*}$ must be modified to incorporate the derivative of the stream function with respect to $K$. The revised definition of $v^{*}$ is

$$
\begin{aligned}
v^{*} & =\frac{v}{(v U(x) / x)^{1 / 2}} \\
& =-\left[\frac{m-1}{m+1}\left(\eta \frac{\partial f(\eta, K)}{\partial \eta}+K \frac{\partial f(\eta, K)}{\partial K}\right)+f(\eta, K)\right]
\end{aligned}
$$

When all other derivatives in $x$ are rewritten to include a $K$ term, the ordinary differential equation given in (12) is replaced by a partial differential equation:

$$
\frac{\partial^{3} f}{\partial \eta^{3}}+f \frac{\partial^{2} f}{\partial \eta^{2}}+\beta\left(1-\left(\frac{\partial f}{\partial \eta}\right)^{2}\right)+K(1-\beta) \frac{\partial}{\partial \eta}\left[\frac{\partial f}{\partial \eta} \frac{\partial f}{\partial K}\right]=0
$$

Equation (20) requires boundary conditions in $K$. As $K$ approaches zero, the no-slip result will be recovered. At large values of $K$, corresponding to the leading edge of the boundary layer, the Navier-Stokes equations, and the continuum hypothesis will have broken down and free-molecular flow will result. This will result in a uniform velocity $u$ and full slip at the wall. The boundary conditions then become

$$
\begin{gathered}
\left.\frac{\partial f}{\partial \eta}\right|_{K \rightarrow \infty}=1 \\
f(K \rightarrow \infty)=\eta
\end{gathered}
$$

These boundary conditions suggest that this formulation may not be accurate at the extreme leading edge of the boundary layer. However, this corresponds to the region where viscous flow theory suggests that the boundary-layer equations are not valid [27]. As in continuum boundary-layer theory, these leading edge errors are assumed to be small and ignored.

\section{B. Energy Equation}

The equation for conservation of energy in a boundary layer with steady flow is given as

$$
u \frac{\partial T}{\partial x}+v \frac{\partial T}{\partial y}=\alpha \frac{\partial^{2} T}{\partial y^{2}}
$$

where $T$ is the local temperature and $\alpha$ is the thermal diffusivity of the gas [29].

The temperature can be nondimensionalized as

$$
T^{*}=\frac{T-T_{w}}{T_{o}-T_{w}}
$$

where $T_{o}$ is the freestream temperature and $T_{w}$ is the surface temperature.

Using the nondimensionalizations used for the fluid flow equations and the nondimensional temperature, the heat equation becomes

$$
\frac{\partial^{2} T^{*}}{\partial \eta^{2}}+\operatorname{Pr} f \frac{\partial T^{*}}{\partial \eta}+K \operatorname{Pr}(1-\beta)\left[\frac{\partial f}{\partial \eta} \frac{\partial T^{*}}{\partial K}+\frac{\partial f}{\partial K} \frac{\partial T^{*}}{\partial \eta}\right]=0
$$

where $P r$ is the Prandtl number of the fluid.

For rarefied flows, a thermal jump condition will occur at the wall:

$$
T_{\text {gas }}-T_{w}=\left.\frac{\lambda}{P r} \frac{2-\sigma_{T}}{\sigma_{T}} \frac{2 \gamma}{\gamma+1} \frac{\partial T}{\partial n}\right|_{\text {wall }}
$$

where $T_{\mathrm{gas}}$ is the temperature of the gas at the wall, $\sigma_{T}$ is the thermal accommodation coefficient, and $\gamma$ is the specific heat ratio [30]. This expression can be nondimensionalized to obtain

$$
T^{*}(\eta=0)=\left.\frac{1}{\operatorname{Pr}} \frac{2-\sigma_{T}}{\sigma_{T}} \frac{2 \gamma}{\gamma+1} \sqrt{\frac{m+1}{2}} \lambda \sqrt{\frac{b}{v}} x^{\frac{m-1}{2}} \frac{\partial T^{*}}{\partial \eta}\right|_{\text {wall }}
$$

If the thermal and momentum accommodation coefficients are assumed to be approximately equal, this expression simplifies to

$$
T^{*}(\eta=0)=\left.\frac{1}{\operatorname{Pr}} \frac{2 \gamma}{\gamma+1} K \frac{\partial T^{*}}{\partial \eta}\right|_{\text {wall }}
$$

At large values of $y$, the temperature will approach the freestream value, giving the boundary condition:

$$
T^{*}(\eta=\infty)=1
$$

At large values of $K$, the temperature jump will become large, and a uniform temperature profile will result, giving the boundary condition:

$$
T^{*}(K \rightarrow \infty)=1
$$

As $K$ approaches zero, the no-slip solution should be recovered.

\section{Similarity to Liquid Slip}

The slip condition in slightly rarefied flows is kinematically similar to the slip condition encountered in liquid flows at the microscale. For the flow of a polar fluid, such as water, over a hydrophobic surface, such as silicon or grapheme, there is evidence from both experiment $[\underline{31}, \underline{32}]$ and molecular dynamics simulations $[\underline{33}, \underline{34}]$ of velocity slip at the surface. However, the existence of this condition, and its relevance to engineering flows, are subjects of considerable controversy [35].

The liquid-slip velocity is written in terms of a slip length 1 and the velocity gradient at the wall:

$$
u_{\text {slip }}=\left.l \cdot \frac{\partial u}{\partial n}\right|_{\text {wall }}
$$

The parameter $K$ used in the fluid flow can be written in terms of the Reynolds number, the angle, and the ratio of the slip length to the position along the surface: 


$$
K=\frac{l}{x} \sqrt{\frac{m+1}{2} \operatorname{Re}_{x}^{1 / 2}}
$$

The appropriate thermal boundary condition for a liquid surface with slip is not clear. Whereas previous researchers have assumed that there is no thermal jump $[18,22]$, there is no clear measurement of this effect, and thermal analysis for liquids with slip is not incorporated in the present work.

\section{Numeric Formulation}

The loss of self-similarity means that the boundary layer must be solved as a partial differential equation instead of an ordinary differential equation. Equations (20) and (25) can be solved using a modified boundary-layer solver. Because large values of $K$ correspond to small values of $x$, the solver begins from large values of $K$ and marches toward a $K$ of zero, going from nonequilibrium flows to equilibrium conditions in the process. This corresponds to the process used in standard boundary-layer codes only in nondimensional coordinates [36].

Equation (20) can be simplified by replacing $\partial f / \partial \eta$ with $f^{\prime}$ :

$$
\frac{\partial^{2} f^{\prime}}{\partial \eta^{2}}+f \frac{\partial f^{\prime}}{\partial \eta}+\beta\left(1-\left(f^{\prime}\right)^{2}\right)+K(1-\beta) \frac{\partial}{\partial \eta}\left[f^{\prime} \frac{\partial f}{\partial K}\right]=0
$$

where $f^{\prime}$, and all its derivatives, are found using central-difference schemes:

$$
\begin{gathered}
f_{i, j}^{\prime}=\frac{f_{i, j+1}-f_{i, j-1}}{2 \Delta \eta}+\mathcal{O}\left((\Delta \eta)^{2}\right) \\
\left.\frac{\partial f^{\prime}}{\partial \eta}\right|_{i, j}=\frac{f_{i, j+1}^{\prime}-f_{i, j-1}^{\prime}}{2 \Delta \eta}+\mathcal{O}\left((\Delta \eta)^{2}\right) \\
\left.\frac{\partial^{2} f^{\prime}}{\partial \eta^{2}}\right|_{i, j}=\frac{f_{i, j+1}^{\prime}-2.0 f_{i, j}^{\prime}+f_{i, j-1}^{\prime}}{(\Delta \eta)^{2}}+\mathcal{O}\left((\Delta \eta)^{2}\right)
\end{gathered}
$$

The mixed derivative term is found using an upwind scheme:

$$
\begin{aligned}
& \frac{\partial}{\partial \eta}\left[f^{\prime} \frac{\partial f}{\partial K}\right]_{i, j}=\frac{1}{\Delta \eta} \frac{1}{\Delta K}\left[f_{i, j}^{\prime}\left(f_{i, j}-f_{i-1, j}\right)\right. \\
& \left.\quad-f_{i, j-1}^{\prime}\left(f_{i, j-1}-f_{i-1, j-1}\right)\right]
\end{aligned}
$$

Combining Eqs. (34- $\underline{37}$ ) into Eq. (33) gives the following expression for $f^{\prime}$ :

$$
\begin{aligned}
f_{i-1, j} & =f_{i, j}+\frac{f_{i, j-1}^{\prime}}{f_{i, j}^{\prime}}\left(f_{i-1, j-1}-f_{i, j-1}\right) \\
+ & \frac{2 \Delta K}{(1-\beta) K f_{i, j}^{\prime}}\left(\frac{f_{i, j+1}^{\prime}-2.0 f_{i, j}^{\prime}+f_{i, j-1}^{\prime}}{\Delta \eta}\right. \\
+ & \left.\frac{f_{i, j}}{4.0}\left(f_{i, j+1}^{\prime}-f_{i, j-1}^{\prime}\right)+\frac{\beta \Delta \eta}{2}\left(1-\left(f_{i, j}^{\prime}\right)^{2}\right)\right)
\end{aligned}
$$

The slip condition is implemented using the following:

$$
f_{i, 1}^{\prime}=\frac{K_{i} f_{i, 2}^{\prime}}{\left(K_{i}+\Delta \eta_{\text {wall }}\right)}+\mathcal{O}\left(\frac{(\Delta \eta)^{2}}{\left(\Delta \eta+K_{i}\right)}\right) \frac{\partial^{2} f^{\prime}}{\partial \eta^{2}}
$$

Equations (14) and (15) provide the other required boundary conditions:

$$
\begin{gathered}
f_{i, 1}=0 \\
f_{i, m}=f_{i, m-1}+\Delta \eta
\end{gathered}
$$

Discretizing the energy equation is simpler. Using centraldifference approximations in $\eta$ and an upwind scheme in $K$, the energy equation becomes

$$
\begin{aligned}
& T_{i-1, j}^{*}=T_{i, j}^{*}+\frac{\left(T_{i, j+1}^{*}-T_{i, j-1}^{*}\right)\left(f_{i, j}-f_{i-1, j}\right)}{2 f_{i . j}^{\prime} \Delta \eta} \\
& +\frac{\Delta K}{f_{i . j}^{\prime} K \operatorname{Pr}(1-\beta)}\left[\frac{\left(T_{i, j+1}^{*}-2.0 T_{i, j-1}^{*}+T_{i, j-1}^{*}\right)}{(\Delta \eta)^{2}}\right. \\
& \left.\quad+\operatorname{Prf}_{i, j} \frac{\left(T_{i, j+1}^{*}-T_{i, j-1}^{*}\right)}{2 \Delta \eta}\right]
\end{aligned}
$$

The wall temperature-jump boundary condition becomes

$$
T_{i, 1}^{*}=\frac{\left(\frac{1}{\operatorname{Pr}} \frac{2 \gamma}{\gamma+1}\right)}{\left(\frac{1}{\operatorname{Pr}} \frac{2 \gamma}{\gamma+1}\right)+\Delta \eta} T_{i, 2}^{*}
$$

The far-field boundary condition is given as

$$
T_{i, m}^{*}=1
$$

For the stagnation-flow case, the momentum and energy equations are solved using a shooting method, just as is done for the no-slip formulation of Falkner-Skan flow.

\section{Fluid Flow Results}

Figures $\underline{2}$ and $\underline{3}$ show the nondimensional wall-slip velocity and wall shear stress as functions of $K$ for half-angles of 15, 30, 45, 60, and $75 \mathrm{deg}$. Previously reported values for a flat plate $(0 \mathrm{deg})[18]$ and a stagnation point (90 deg) [21] are included for reference. Because this formulation may be valid for liquid flows with slip and values of $K$ greater than one, values of $K$ greater than one are shown. These results suggest that slip effects become important at values of $K$ less than 1.0, including regions where the continuum assumption is valid for gases. They also suggest that the relative importance of slip effects increases with increasing wedge angle.

Figure 3 shows that for the flat plate and 15-deg wedge, the maximum shear stress is encountered not at the equilibrium condition but in slightly rarefied flow. This local minimum disappears as the wedge half-angle increases. This surprising result agrees with earlier analysis [18] of a flat-plate slip flow.

The velocity profiles for $u^{*}$ and $v^{*}$ as a function of $\eta$, for values of $K$ of less than 1.0, and angles of 0,30 , and $60 \mathrm{deg}$, are shown as Figs. 4 and 5 . These results confirm the loss of self-similarity in the flow. For flow in the $x$ direction, these results show wall slip. For flow in the $y$ direction, these results show an increase in the tangential velocity, pulling mass, momentum, and energy away from the wall.

In addition to the shear stress, the boundary-layer thickness, velocity thickness, and momentum thickness are quantities of interest in boundary-layer flows [27]. The boundary-layer thickness $\eta_{99}$ is defined as the point where the velocity is equal to $99 \%$ of the freestream velocity, as described in Eq. (45):

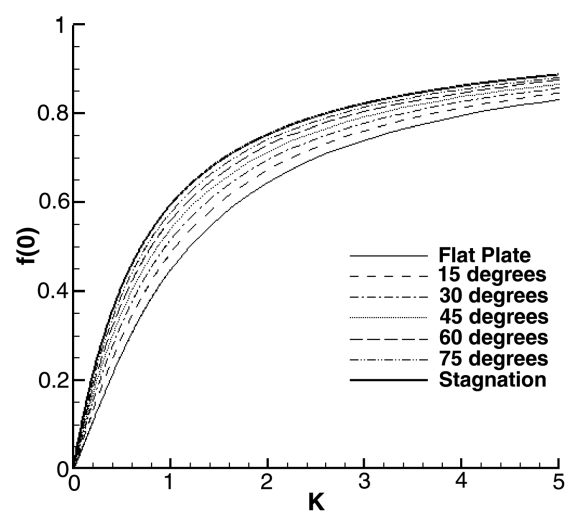

Fig. 2 Nondimensional wall velocity as a function of $K$. 


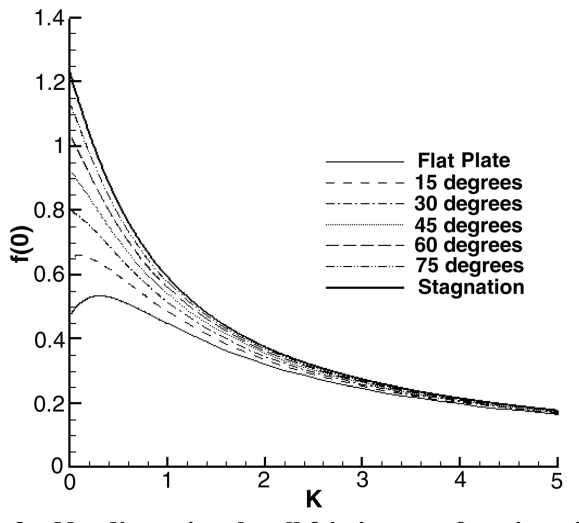

Fig. 3 Nondimensional wall friction as a function of $K$.

$$
\left.\frac{\partial f}{\partial n}\right|_{\eta=\eta_{99}}=0.99
$$

The velocity thickness $\delta^{*}$ and momentum thickness $\theta$ are defined as

$$
\begin{gathered}
\delta^{*}=\int_{y=0}^{\infty} \frac{U(x)-u(x, y)}{U(x)} \mathrm{d} y=\int_{\eta=0}^{\infty}\left(1-f^{\prime}(\eta)\right) \mathrm{d} \eta \\
\theta=\int_{y=0}^{\infty} \frac{u(x, y)(U(x)-u(x, y))}{U^{2}(x)} \mathrm{d} y=\int_{\eta=0}^{\infty} f^{\prime}(\eta)\left(1-f^{\prime}(\eta)\right) \mathrm{d} \eta
\end{gathered}
$$

Figures $\underline{6}-\underline{8}$ show the boundary-layer thickness, velocity thickness, and momentum thickness as functions of $K$ for half-angles of 0 ,

$15,30,45,60,75$, and $90 \mathrm{deg}$. These results show that the boundarylayer thickness may decrease by $50 \%$ or more. The velocity thickness and momentum thickness may decrease by even larger amounts at high values of $K$. These results appear to be relatively independent of wedge half-angle.

Researchers studying the flat-plate boundary layer use a Reynolds number based on velocity thickness to predict the transition to turbulence:

$$
\operatorname{Re}_{\delta}=\rho U \delta^{*} / \mu
$$

When $R e_{\delta}$ approaches 520, the boundary layer becomes unstable, a first step in the transition to turbulence [27]. Because the effect of slip is to decrease the velocity thickness, rarefaction may delay the onset of turbulence.

These results also agree qualitatively with trends observed computational fluid dynamics simulations [37] and measurements [38] of hypersonic flow over a flat plate. The results showed that the skin friction and boundary-layer thickness decreased as the momentum accommodation coefficient decreased, which corresponds to an increase in $K$.

A final quantity of interest is the total shear force in the $x$ direction. The shear force on one side of the wedge can be expressed in terms of a nondimensional viscous drag coefficient:

$$
C_{D, \text { visc }}=\frac{F_{\mathrm{visc}}}{0.5 \cdot L \cdot \rho \cdot U(L)^{2}}=\frac{1}{0.5 \cdot L \cdot \rho \cdot U(L)^{2}} \int_{0}^{L} \tau(x) \mathrm{d} x
$$

For the no-slip case of Falkner-Skan flow, the drag coefficient will be

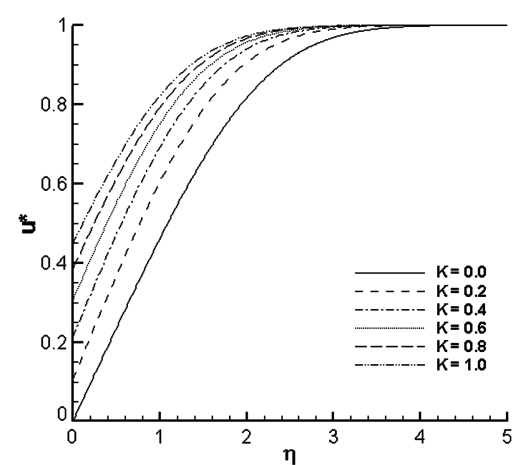

a) Flat plate

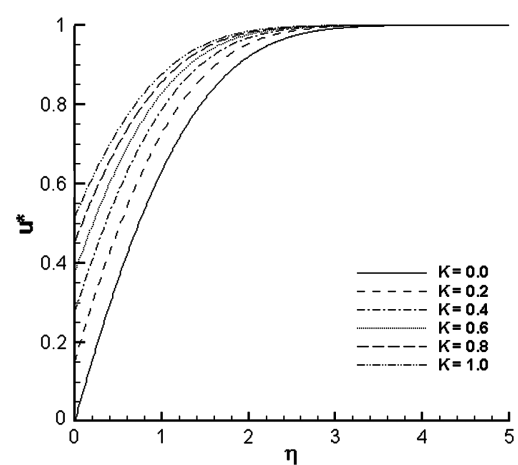

b) 30 degrees

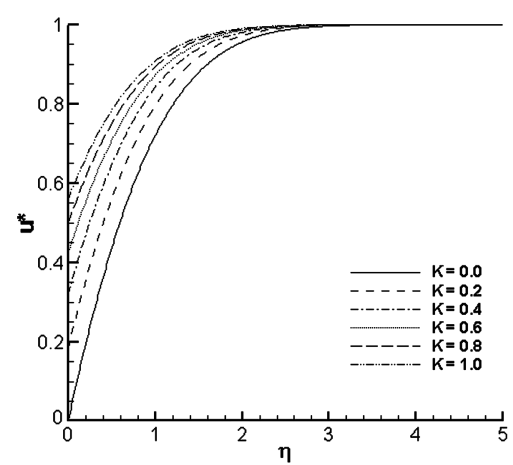

c) 60 degrees

Fig. 4 Nondimensional $x$-velocity profiles for a flat plate; 30, and $60 \mathrm{deg}$.

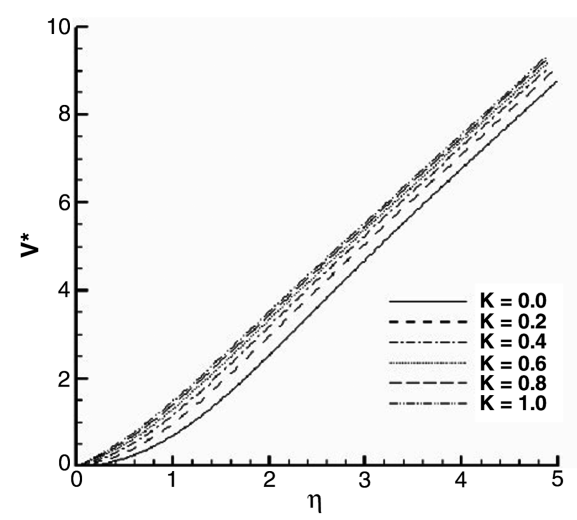

a) Flat plate

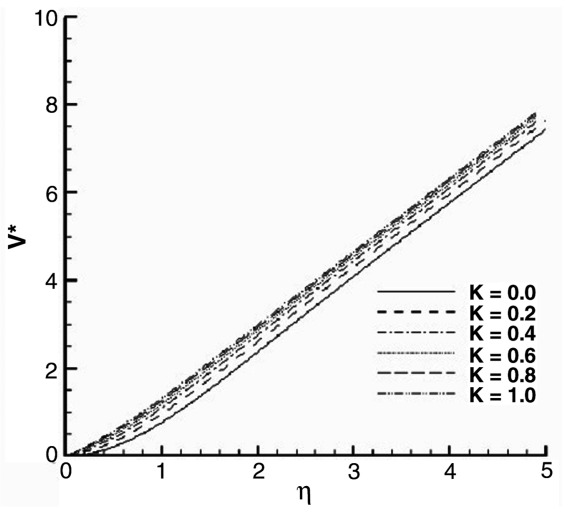

b) 30 degrees

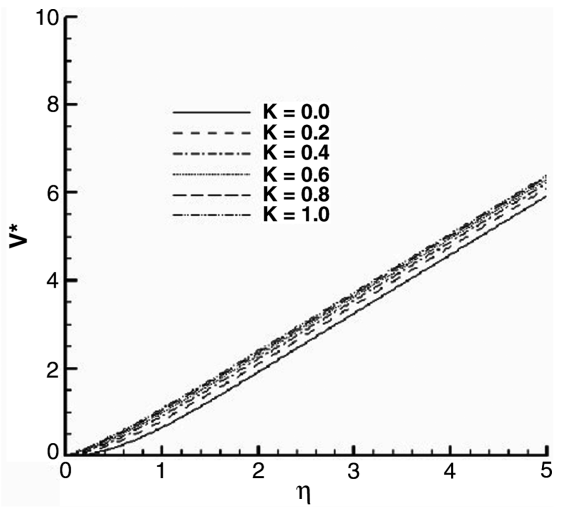

c) 60 degrees

Fig. 5 Nondimensional $y$-velocity profiles for a flat plate; 30, and $60 \mathrm{deg}$. 


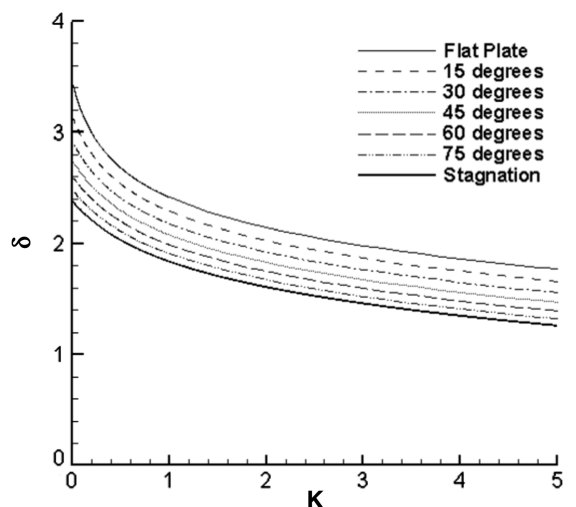

Fig. 6 Boundary-layer thickness as a function of $K$.

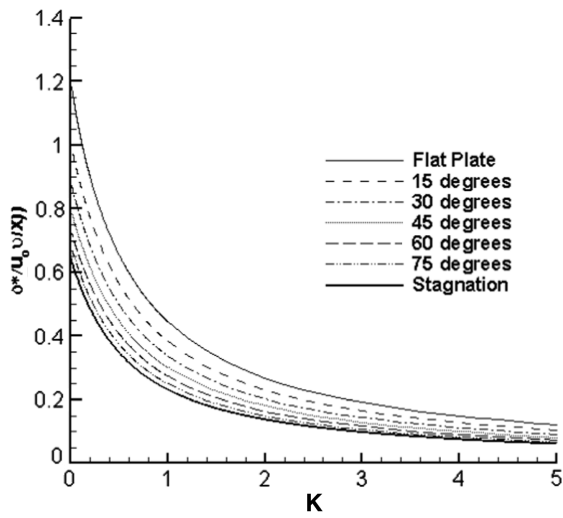

Fig. 7 Velocity thickness as a function of $K$.

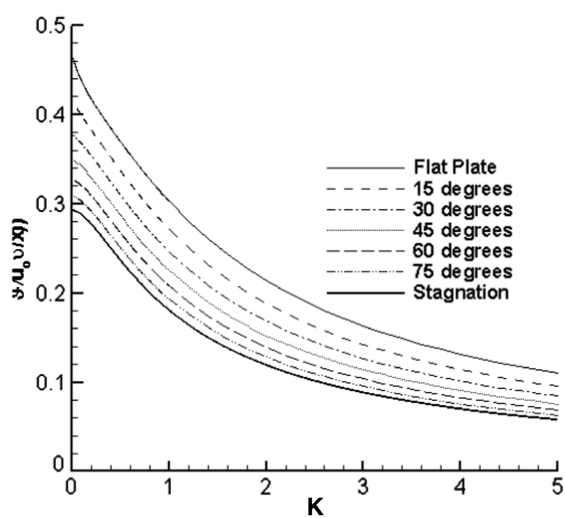

Fig. 8 Momentum thickness as a function of $K$.

$$
C_{D, \mathrm{visc}}=\left(\frac{4}{3 m+1}\right)\left(\frac{m+1}{2}\right)^{0.5} \frac{f^{\prime \prime}(0)}{R e_{L}^{0.5}}
$$

For the slip case, the drag coefficient will be

$$
C_{D, \text { visc }}=\frac{1}{R e_{L}^{1 / 2}}\left(\frac{4}{1-m}\right)\left(\frac{m+1}{2}\right)^{0.5} K^{\frac{3 m+1}{1-m}} \int_{K(L)}^{\infty} K^{\frac{2 m+2}{m-1}} f^{\prime \prime}(0) \mathrm{d} K
$$

The viscous drag coefficient multiplied by the square root of the Reynolds number as a function of $K(L)$ is shown as Fig. 9. At the limit of $K$ equal to zero or a Knudsen number of zero, the no-slip values are used. At zero angle of attack, the results show an increase in drag for slightly rarefied flows and a decrease as the flow becomes more rarefied consistent with previous analysis [18]. As the angle of attack increases, the results shift and the drag decreases for all rarefied flows. This is consistent with previous analysis of rarefied stagnation-point flows, where the shear stress decreases with rarefaction $[\underline{21}, \underline{22}]$.

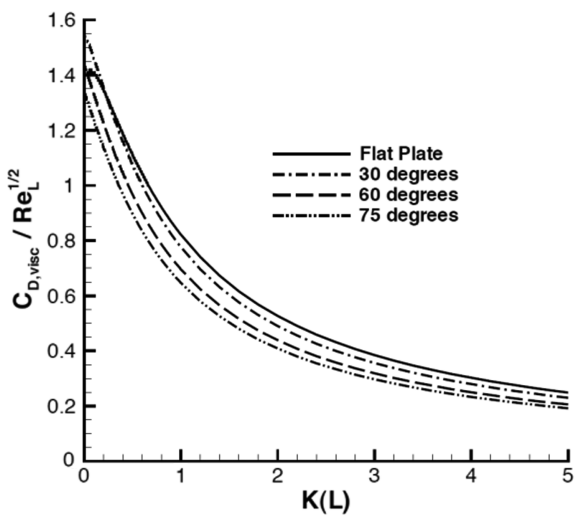

Fig. 9 Drag coefficient as a function of $K(L)$ for a flat plate; 30, 60, and 75 deg.

These results also agree qualitatively with trends observed computational fluid dynamics simulations [37] and measurements [38] of hypersonic flow over a flat plate. The results showed that the skin friction and boundary-layer thickness decreased as the momentum accommodation coefficient decreased, which corresponds to an increase in $K$.

\section{Heat Transfer Results}

The heat transfer is computed for a specific heat ratio of 1.4, representing a diatomic gas. Three representative Prandtl numbers, $0.7,1.0$, and 1.4 , are used to determine the effect of changing the Prandtl number of the gas. Figure 10 shows the gas temperature at the wall as a function of $K$ for wedge half-angles of 0,30 , and $60 \mathrm{deg}$ for a diatomic gas. The increase in gas temperature at the wall because of rarefaction effects is largest for the flat-plate case and decreases with increasing wedge half-angle. As expected from Eq. (26), the temperature jump decreases with increasing Prandtl number.

Figure 11 shows the nondimensionalized wall heat transfer as a function of $K$ for wedge half-angles of 0,30 , and $60 \mathrm{deg}$ for diatomic gases. These results show a local maximum in the heat transfer at values of $K$ of less than one for 0,30 , and $60 \mathrm{deg}$. This result may be considered to be analogous to the local maximum in shear stress encountered earlier. The maximum moves to lower values of $K$ with decreasing Prandtl numbers and with increasing wedge half-angles. The local maximum disappears entirely in the stagnation-flow condition.

The difference in heat transfer rates between slip-flow and nonslip flow cases increases with increasing Prandtl number. The increase in heat transfer appears to be slightly higher for the diatomic cases. Overall, the heat transfer increases by as much as $50 \%$ or decreases by as much as $80 \%$, from nonequilibrium values, depending on the flow conditions. These results agree qualitatively with experimental measurements taken on cylinders at low speeds [39].

The average-heat transfer coefficient over a surface of the wedge can be expressed using an average Nusselt number:

$$
\overline{N u}_{L}=\frac{\bar{h} \cdot L}{k}=\frac{1}{k} \int_{0}^{L} h(x) \mathrm{d} x
$$

For the no-slip case of Falkner-Skan flow, the average Nusselt number will be

$$
\overline{N u}_{L}=\left.\left(\frac{2}{m+1}\right)^{0.5} R e_{x}^{0.5} \frac{\mathrm{d} T}{\mathrm{~d} \eta}\right|_{\eta=0}
$$

For the slip case, the average Nusselt number will be

$$
\overline{N u}_{L}=\left.\left(\frac{2}{1-m}\right)\left(\frac{m+1}{2}\right)^{0.5} R e_{x}^{0.5} K^{\frac{m+1}{1-m}} \int_{K(L)}^{\infty} K^{\frac{2}{m-1}} \frac{\mathrm{d} T}{\mathrm{~d} \eta}\right|_{\eta=0} \mathrm{~d} K
$$




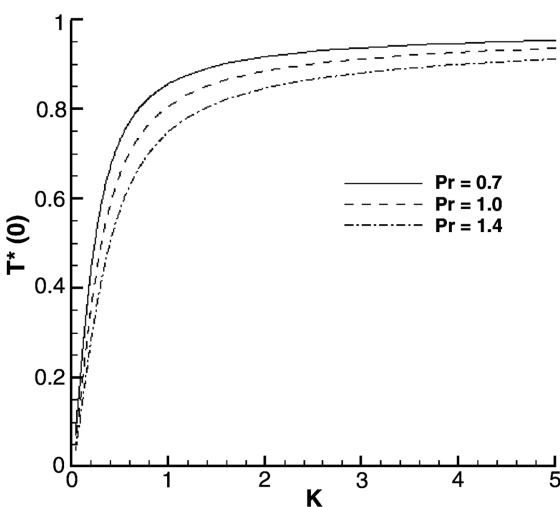

a) Flat plate

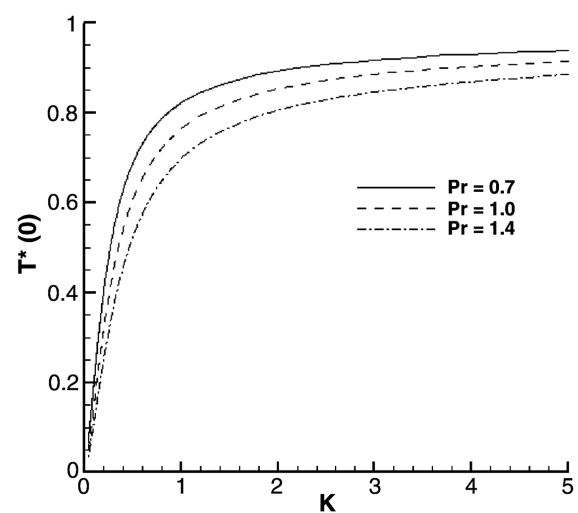

b) 30 degrees

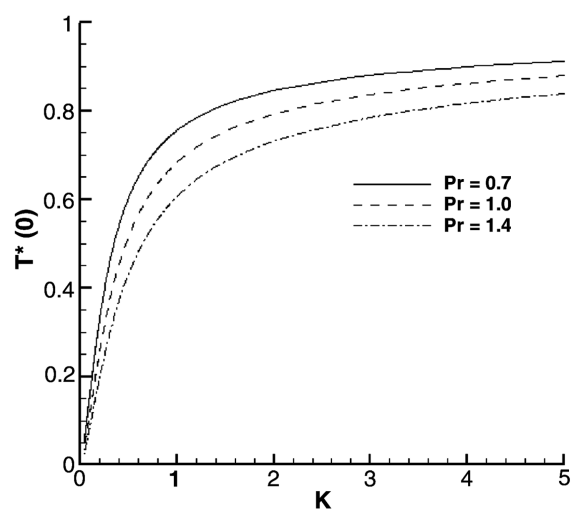

c) 60 degrees

Fig. 10 Nondimensional wall temperature as a function of $K$ and $\gamma=1.4$ for a flat plate; 30, and $60 \mathrm{deg}$.

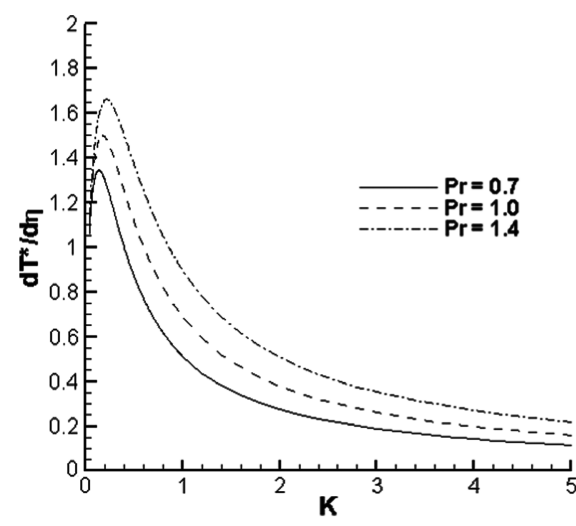

a) Flat plate

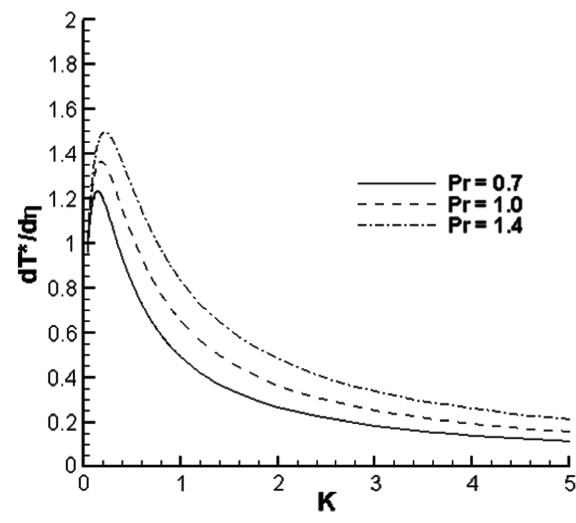

b) 30 degrees



c) 60 degrees

Fig. 11 Nondimensional heat transfer as a function of $K$ and $\gamma=1.4$ for a flat plate; 30, and $60 \mathrm{deg}$.

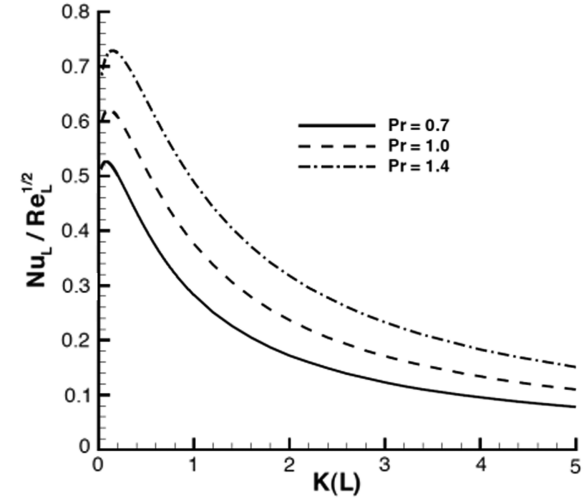

a) Flat plate

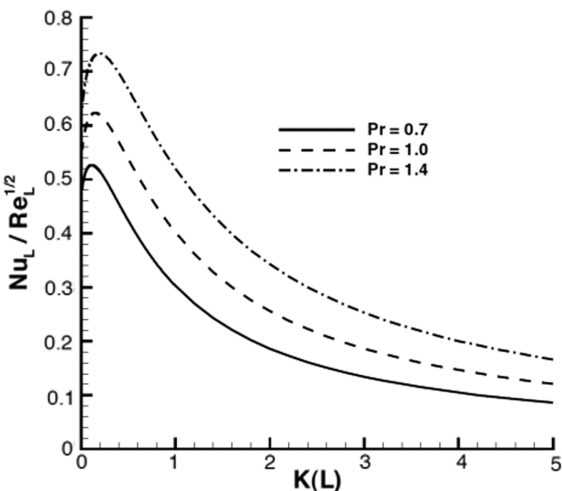

b) 30 degrees

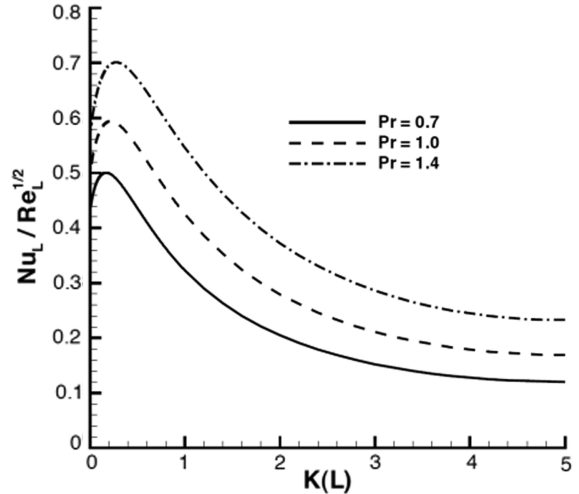

c) 60 degrees

Fig. 12 Average Nusselt number as a function of $K_{L}$ and $\gamma=1.4$ for a flat plate; 30, and $60 \mathrm{deg}$.

The average Nusselt divided by the square root of the Reynolds number as a function of $K(L)$ is shown as Fig. 12. At the limit of $K$ equal to zero, or a Knudsen number of zero, the no-slip values are used. Because of the maximum values of local heat transfer seen at low Knudsen numbers, all of these plots show a maximum value for the average heat transfer at a value of $K$ between 0.1 and 0.5 . This is consistent with previous results for flat-plate boundary layers [18]. As the angle of attack increases, the results shift, and the drag decreases for all rarefied flows. This is consistent with previous analysis of rarefied stagnation-point flows, where the shear stress decreases with rarefaction $[21,22]$.

\section{Conclusions}

Slip flow over a wedge was analyzed incorporating a slip boundary condition. The nondimensional governing equations used to compute the flow in the no-slip condition were modified to allow for the loss of self-similarity that accompanies the slip boundary condition. 
Numeric solution of the modified boundary-layer equations showed a local increase in skin friction under slightly rarefied conditions, and then a decrease in skin friction as the flow became more rarefied. This effect disappeared as the angle of the wedge increased. The relative decrease in skin friction became larger as the wedge angle increased.

Use of a modified temperature-jump equation allowed the heat transfer to be calculated for the wedge. These results showed an increase in wall temperature and a decrease in heat transfer because of rarefaction effects. These effects were weakly dependent on the specific heat ratio of the gas and strongly dependent on the Prandtl number and angle of the wedge.

\section{Acknowledgments}

The authors wish to thank the Louisiana Optical Network Initiative for computing support and Matthew McNenly for useful technical discussions.

\section{References}

[1] Hutchins, D. K., Harper, M. H., and Felder, R. L., "Slip Correction Measurements for Solid Spherical Particles by Modulated Dynamic Light Scattering," Aerosol Science and Technology, Vol. 22, No. 2, 1995, pp. 202-218.

doi:10.1080/02786829408959741

[2] Braun, R. D., Wright, H. S., Croom, M. A., Levine, J. S., and Spencer, D. A., "Design of the ARES Mars Airplane and Mission Architecture," Journal of Spacecraft and Rockets, Vol. 43, No. 5, 2006, pp. 10261034. doi: $10.2514 / 1.17956$

[3] Sun, Q., Boyd, I. D., and Candler, G. V., "Numerical Simulation of Gas Flow Over Microscale Airfoils," Journal of Thermophysics and Heat Transfer, Vol. 16, No. 2, 2002, pp. 171-179. doi: $10.2514 / 2.6681$

[4] Mueller, T. J., and DeLaurier, J. D., "Aerodynamics of Small Vehicles," Annual Review of Fluid Mechanics, Vol. 35, Jan. 2003, pp. 89-111. doi:10.1146/annurev.fluid.35.101101.161102

[5] Lin, T. C., and Schaaf, S. A., "Effect of Slip on Flow Near a Stagnation Point and in a Boundary Layer," NACA TN-2568, 1951.

[6] Maslen, S. H., "Second Approximation to Laminar Compressible Boundary Layer on Flat Plate in Slip Flow," NACA TN-2818, 1952.

[7] Nonweiler, T., "The Laminar Boundary Layer in Slip Flow," College of Aeronautics Cranfield Univ., Rept. 62, Cranfield, England, U.K., 1952.

[8] Reddy, K. C., "Thermal Boundary Layer in Slip Flow Regime," AIAA Journal, Vol. 1, No. 10, 1963, pp. 2396-2398. doi: $10.2514 / 3.2078$

[9] Kogan, M. N., Rarefied Gas Dynamics, Plenum, New York, 1969.

[10] Hasimoto, H., "Boundary-Layer Slip Solutions for a Flat Plate," Journal of the Aeronautical Sciences, Vol. 25, No. 1, 1958, pp. 68-69.

[11] Pozzi, A., "Similar Solutions in Boundary Layer Slip Flow," AIAA Journal, Vol. 1, No. 5, 1963, pp. 1219-1219.

[12] Wuest, W., "Boundary Layers in Rarefied Gas Flow," Progress in Aerospace Sciences, Vol. 8, 1967, pp. 295-352. doi:10.1016/0376-0421(67)90006-1

[13] Maslen, S. H., "Second-Order Effects in Laminar Boundary Layers," AIAA Journal, Vol. 1, No. 1, 1963, pp. 33-40. doi: $10.2514 / 3.1462$

[14] Van Dyke, M., "Higher Order Boundary Layer Theory," Annual Review of Fluid Mechanics, Vol. 1, Jan. 1969, pp. 265-292. doi:10.1146/annurev.fl.01.010169.001405

[15] Gombosi, T. A., Gaskinetic Theory, Cambridge Univ. Press, Cambridge, England, U.K., 2002.

[16] Ho, C. M., and Tai, Y.-C., "Micro-Electro-Mechanical-Systems (MEMS) and Fluid Flows," Annual Review of Fluid Mechanics, Vol. 30, Jan. 1998, pp. 579-612. doi:10.1146/annurev.fluid.30.1.579

[17] Gad-el-Hak, M., "The Fluid Mechanics of Microdevices: The Freeman Scholar Lecture," Journal of Fluids Engineering, Vol. 121, No. 1, 1999, pp. 5-33. doi: $10.1115 / 1.2822013$
[18] Martin, M. J., and Boyd, I. D., "Momentum and Heat Transfer in a Laminar Boundary Layer with Slip Flow," Journal of Thermophysics and Heat Transfer, Vol. 20, No. 4, 2006, pp. 710-719. doi:10.2514/1.22968

[19] Arkilic, E. B., Schmidt, M. A., and Breuer, K. S., "Gaseous Slip Flow in Long Microchannels," Journal of Microelectromechanical Systems, Vol. 6, No. 2, 1997, pp. 167-178. doi: $10.1109 / 84.585795$

[20] Harley, J. C., Huang, Y. F., Bau, H. H., and Zemel, J. N., "Gas Flow In Micro-Channels," Journal of Fluid Mechanics, Vol. 284, 1995, pp. 257-274. doi:10.1017/S0022112095000358

[21] Tamada, K., "Stagnation-Point Flow of a Rarefied Gas," Journal of the Physical Society of Japan, Vol. 22, No. 5, 1967, pp. 1284-1295. doi:10.1143/JPSJ.22.1284

[22] Wang, C. Y., "Stangation Flows with Slip: Exact Solutions of the Navier-Stokes Equations," Zeitschrift für Angewandte Mathematik und Physik, Vol. 54, No. 1, 2003, pp. 184-189. doi:10.1007/PL00012632

[23] Martin, M. J., and Boyd, I. D., "Stagnation Point Flow near the Continuum Limit," AIAA Journal, Vol. 47, No. 1, 2009, pp. 283-285. doi:10.2514/1.39789

[24] Cao, K., and Baker, J., "Natural Convection over Vertical Plates: LocalNonsimilarity Slip Flow Solutions," Journal of Thermophysics and Heat Transfer, Vol. 23, No. 3, 2009, pp. 522-532. doi: $10.2514 / 1.37418$

[25] Blasius, H., "Grezzschichten in Flüssigkeiten mit Kleiner Reibung," Zeitschrift für Mathematik und Physik, Vol. 56, No. 1, 1908, pp. 1-37.

[26] Falkner, V. M., and Skan, S. W., "Solutions of the Boundary-Layer Equations," Philosophical Magazine, Vol. 7, No. 12, 1931, pp. 865896.

[27] Schlichting, H., and Gersten, K., Boundary Layer Theory, McGrawHill, New York, 2000.

[28] Maxwell, J. C., "On Stresses in Rarefied Gases Arising from Inequalities of Temperature," Philosophical Transactions of the Royal Society of London, Vol. 170, Jan. 1879, pp. 231-256. doi:10.1098/rstl.1879.0067

[29] Incropera, F. P., and DeWitt, D. P., Fundamentals of Heat and Mass Transfer, Wiley, New York 2001.

[30] Smoluchowski von Smolan, M., "Über Wärmeleitung in Verdünnten Gasen," Annalen der Physik und Chemie, Vol. 300, No. 1, 1898, pp. 101-130. doi:10.1002/andp. 18983000110

[31] Watanabe, W., Yanuar, and Mizunama, H., "Slip of Newtonian Fluids at Solid Boundary," JSME International Journal Ser. B, Vol. 41, No. 3 , 1998, pp. 525-529.

[32] Tretheway, D. C., and Meinhart, C. D., "Apparent Fluid Slip at Hydrophobic Microchannel Walls," Physics of Fluids, Vol. 14, No. 3, 2002, pp. L9-L12. doi: $10.1063 / 1.1432696$

[33] Thompson, P. A., and Troian, S. M., "A General Boundary Condition for Liquid Flow at Solid Surfaces," Nature, Vol. 389, Sept. 1997, pp. 360-326.

[34] Walther, J. H., Werder, T., Jaffe, R. L., and Koumoutsakos, P., "Hydrodynamic Properties of Carbon Nanotubes," Physical Review E (Statistical Physics, Plasmas, Fluids, and Related Interdisciplinary Topics), Vol. 69, No. 6, 2004, Paper 062201.

[35] Stone, H. A., Stroock, A. D., and Ajdari, A., "Engineering Flows in Small Devices," Annual Review of Fluid Mechanics, Vol. 36, Jan. 2004, pp. 381-411. doi:10.1146/annurev.fluid.36.050802.122124

[36] White, F., Viscous Fluid Flow, 2nd ed., McGraw-Hill, New York, 1991, pp. 276-282.

[37] Lofthouse, A. J., and Boyd, I. D., "Hypersonic Flow over a Flat Plate: CFD Comparison with Experiment," AIAA Paper 2009-1315, Jan. 2009.

[38] Cecil, E., and McDaniel, J. C., "Planar Velocity and Temperature Measurements in Rarefied Hypersonic Flow Using Iodine LIF," AIAA Paper 2005-4695, June 2005.

[39] Baldwin, L., Sandborn, V., and Laurence, J., "Heat Transfer from Transverse and Yawed Cylinders in Continuum, Slip, and Free Molecule Air Flows," Journal of Heat Transfer, Vol. 82, No. 2, 1960, pp. $77-88$. 